\title{
Cyclic GMP-gated channels are co-localized with a population of Interstitial cells, but not with neuronal NOS, in the rat urethra
} Marta González, Gonzalo Costa, Ángeles García-Pascual and Domingo Triguero*

Address: Department of Physiology, Veterinary School, Complutense University, 28040 Madrid, Spain

Email: Domingo Triguero* - dtriguer@vet.ucm.es

* Corresponding author

from 2nd International Conference of cGMP Generators, Effectors and Therapeutic Implications Potsdam, Germany, 10-12 June, 2005

Published: 16 June 2005

BMC Pharmacology 2005, 5(Suppl I):P2I doi:I0.II86/|47|-22I0-5-SI-P2I

The present study further analyse the presence of cyclic nucleotide-gated (CNG) channels and its involvement in the nitrergic urethral relaxation. Our functional results confirm and extend to the rat those previously found in the sheep urethra [1]. Moreover, rat urethra is even more sensitive to the effect of L-cis-diltiazem, a selective inhibitor of CNG channels. Double-labelling immunofluores- cence was performed on fixed sections $(30 \mu \mathrm{m})$ of rat urethra to visualize CNG1 subtype channels (goat anti-rat CNG1//FITC-conjugated donkey anti-goat) and its colocalization with either neuronal NOS- (rabbit anti-rat nNOS//Rhodamine-conjugated donkey anti-rabbit) or vimentin- (mouse anti-vimentin//Rhodamine-conjugated donkey anti-mouse) positive cells. Micrographs of the

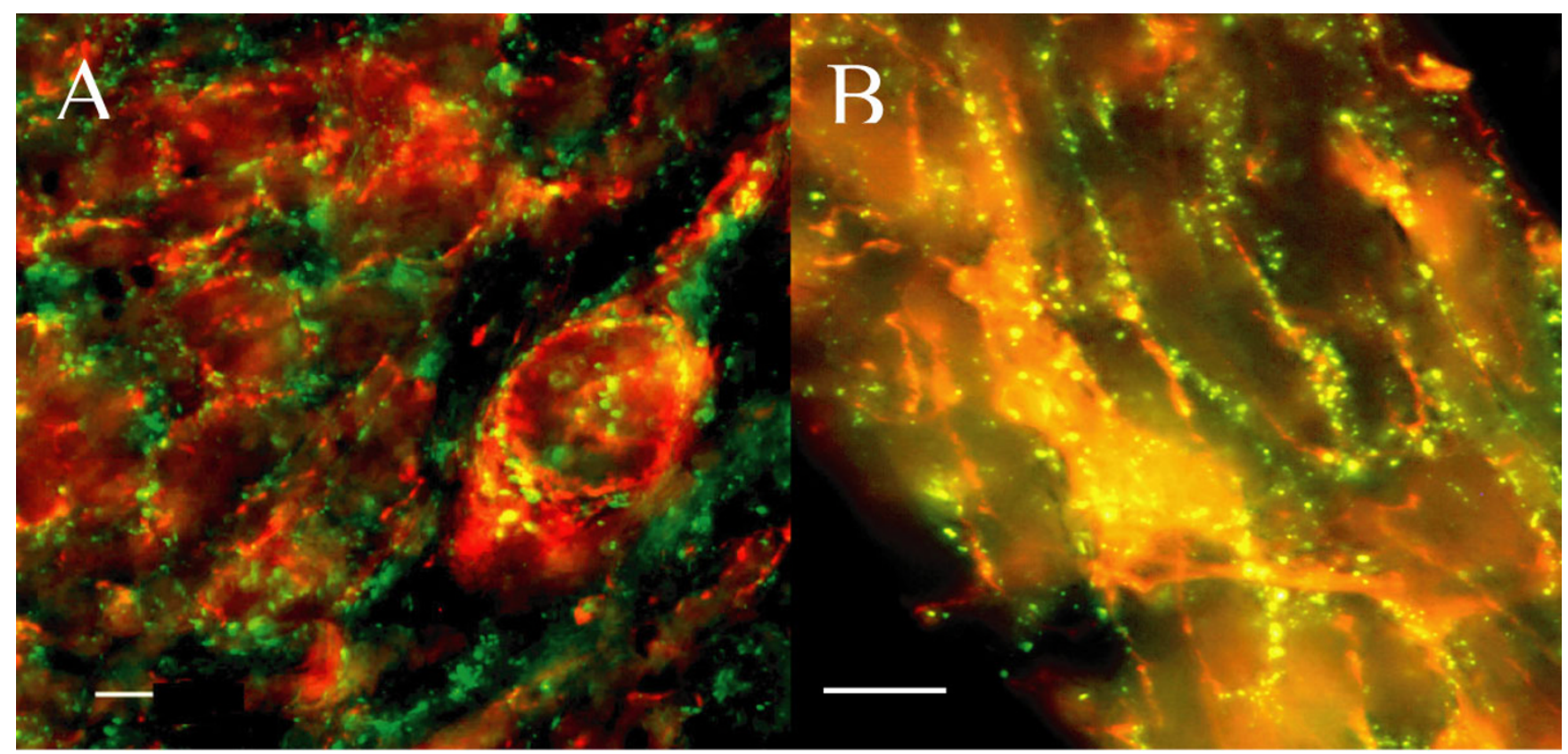

Figure I

Sections of rat urethra showing CNGI-channels immunoreactivity (green) and its relationship with either nNOS (A, red) or vimentin-immunoreactivity (B, red). Scale bar: $25 \mu \mathrm{m}$. 
same field were taken, with separate filters, with an Olympus fluorescence microscope equipped with a cooled CCD camera, and then superimposed by using image analysis software. CNG1-immunoreactivity was present in the muscular layer of rat urethra as a punctuate network surrounding bundles of smooth muscle cells (Figure 1A and 1B, green). Neuronal NOS-positive fibres (Fig 1A, red) were seen as a similar network in the vicinity of CNG1immunoreactivity, but being sparsely overlapped. However, a high degree of co-localization was evident between CNG1- and vimentin-immunoreactivity (Fig 1B, red). This overlapping was specially conspicuous in vimentin-positive spindle shaped cells with long and thin cellular processes that run parallel to smooth muscle cells. These cells have been described as interstitial cells (IC) given its similarity to Cajal's IC at the intestine. By other hand, IC in the sheep urethra have been found to show a very significant increase in cGMP-immunoreactivity when urethral strips were subjected to electrical field stimulation of nitrergic nerves (A, García-Pascual et al, in preparation). Taken together, our present results led us to suggest that a network of IC could be modulating urethral smooth muscle relaxation by a yet undefined mechanism, although it seems likely that ionic events initiated by activation of CNG channels could be involved, subsequently to the rise of cGMP levels triggered by NO released from the adjacent nitrergic nerves.

Supported by grants from Fondo de Investigaciones Sanitarias (FIS 00/0425) and Ministerio de Ciencia y Tecnología (BFI2003-04082-C02-01), Spain.

\section{References}

I. Triguero D, González M, García-Pascual A, Costa G: Atypical relaxation by scorpion venom in the lamb urethral smooth muscle involves both NO-dependent and -independent responses. Naunyn-Schmiedeberg's Arch Pharmacol 2003, 368:151-159.

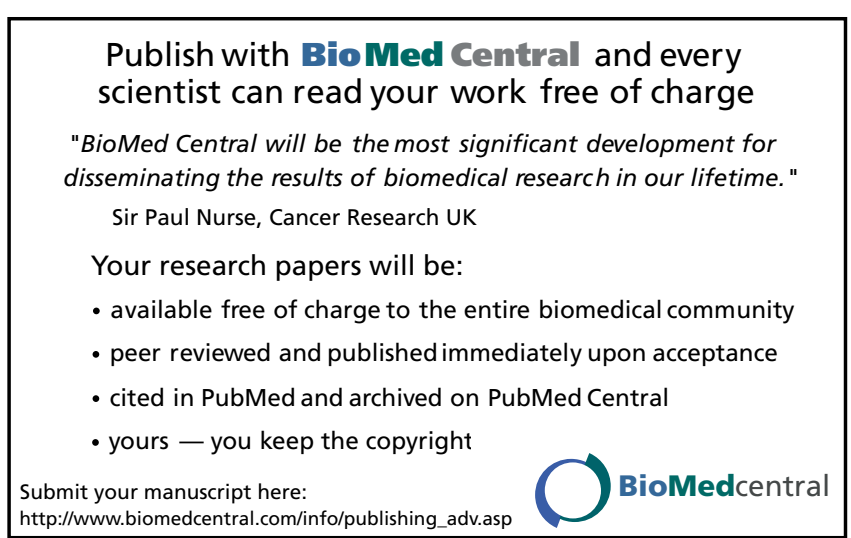

Page 2 of 2

(page number not for citation purposes) 